\title{
Effect of Different Diets with Sugar-Peptide Beverage on Oxidation of Cathohvdrate and Lipid after Exercise
}

\author{
Ronghua Liu* \\ Chang'an District of Xi'an Chang'an Road No. 199 Shaanxi Province, Shaanxi Normal University, China
}

\begin{abstract}
Human tests show that the long-term high-fat diet adaptation can increase fat oxidation in the exercise significantly than high-carbohydrates. Fewer studies focus on single high fat diet on glucose and lipid metabolism and the change of fat oxidation. This test attempts to use respiratory quotient as an effective indicator to reflect the body's metabolism of glucose and lipid and evaluate the oxidation of different dietary conditions in order to provide a direct basis for people's reasonable diet.
\end{abstract}

Keywords: Different diets, Exercise, Oxidation of cathohvdrate, Oxidation of lipid, Sugar-peptide beverage.

\section{INTRODUCTION}

In recent years, residents have changed a lot of food choices with economic development, people's income has increased and market food has become enriched. Dietary patterns tend to change unhealthy to the high-fat, high-energy and low-grain due to increase in consumption of meat, oils and fats and subsequently a fall in consumption of cereals, pulses and vegetable [1]. This will adversely affect human health. The Chinese nutrition society has combined our actual dietary patterns and developed dietary with suitable fat intake for adults (AIS) within the total energy intake in $20 \% \sim 30 \%$. Therefore, it is the low-fat diet with the requirement below $20 \%$ and the high-fat diet with the requirement above $30 \%$. The dietary lipids (fat, cholesterol and fatty acids) are closely related to health. As the living standards improve and the market supply of animal food and vegetable oil is becoming richer, residents are registered with increase in dietary fat intake [2]. A number of residents in economically developed regions in China are particularly evident of this fact as the fat percentage of their total energy intake has exceeded $30 \%$. Therefore, how to control fat and cholesterol intake and adjust the fatty acid composition to reduce obesity, cardio-cerebral vascular disease, cancer and other chronic diseases have become the key issues in the field of nutrition [3].

In the $20^{\text {th }}$ century, there have been several kinds of weight-reduction diets. Such diets advocate free intake of foods that are rich in protein and fat, but refuse foods with high carbohydrates. One of them is Marri diet in 1949. The following is Prudent diet, Gordon diet, Donalds diet, Yudkin diet and other similar weight-loss diets. This dietary law is based on the theory of carbohydrates, particularly sugar which has a lower satiety value and cause an "artificial taste". People are interested in dietary supplementation with dietary fiber. The researchers believe that diets which are rich in dietary fiber can reduce the total energy intake and

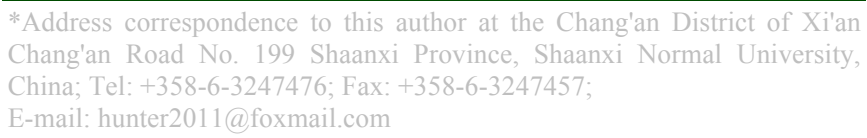

help control the weight without detailed numerical calculation of dietary energy. Most popular diets currently aim to limit dietary fats. Many nutritionists and other health professionals have advocated to permanently maintaining a low-fat diet in order to lose weight and retain their weight on the low [4]

We generally use nutrition to measure dietary standards. In terms of energy research, the FAO determines the minimum calorific value of food to be $2300 \mathrm{kcal}$ which is relatively comfortable of $2600 \mathrm{kcal}$ [5]. The World Health Organization (WHO) has put forward that the real intake of fat should account $15 \%$ to $30 \%$ for the total dietary energy. In terms of protein intake, it is recommended that the animal protein account for 62.50 in United States food pyramid. Physical activity can enhance the ability to regulate blood sugar and insulin sensitivity and would also help to reduce the incidence of CVD. Once we stop exercising, the benefits of exercise will soon disappear. The acute effects of exercise on body may improve the glucose tolerance and insulin resistance which is an important part of critical points. Therefore studies of acute exercise are also essential.

The rest of the paper is organized as follows. In Section 2 , research methods are summarized briefly. In Section 3, experimental results are described. In Section 4, experiments results are presented and the results are discussed (Missing information). Finally, a conclusion is provided in Section 5.

\section{RESEARCH METHODS}

\subsection{Subject}

There are eight healthy adult males who volunteered for the experiment met the following conditions: non-smokers, age 24-27, weight normal, no metabolism sex disease and diabetes, and often participate in running and other physical activities. The Subjects must have movement endurance level $\mathrm{w}$ to complete the test by fulfilling experiment requirements as presented in Table 1 . 
Table 1. Basic situation of the subjects.

\begin{tabular}{|c|c|c|c|c|c|}
\hline $\begin{array}{c}\text { Age } \\
(\text { year})\end{array}$ & $\begin{array}{c}\text { Height } \\
(\mathbf{c m})\end{array}$ & $\begin{array}{c}\text { Weight } \\
(\mathbf{k g})\end{array}$ & $\begin{array}{c}\text { BMI } \\
\left(\mathbf{k g} / \mathbf{m}^{\mathbf{2}}\right)\end{array}$ & $\begin{array}{c}\text { Hrmax } \\
(\mathbf{b e a t s} / \mathbf{m i n})\end{array}$ & $\begin{array}{c}\text { VO } \\
(\mathbf{m} \mathbf{m a x} \\
(\mathbf{m i n})\end{array}$ \\
\hline \hline $25 \pm 0.3$ & $174.1 \pm 2.2$ & $67.7 \pm 2.8$ & $22.3 \pm 0.6$ & $184.0 \pm 2.8$ & $4.05 \pm 0.2$ \\
\hline
\end{tabular}

Table 2. Eating design order.

\begin{tabular}{|c|c|c|c|c|}
\hline & Week 1 & Week 2 & Week 3 & Week 4 \\
\hline \hline Monday & CC & FC & CP & FP \\
\hline Tuesday & FC & CP & CC & FC \\
\hline Wednesday & CP & FP & FC & CP \\
\hline Thursday & FP & CC & FP & \\
\hline
\end{tabular}

\subsection{The Preliminary Experiment}

We use the power car to do the movement tests. Cardiopul-monary exercise tests for measuring oxygen uptake and res-piratory exchange ratio. Telemetry heart rat monitor records the heart rate. The lab environment is as follows: temperature $19-230 \mathrm{C}$, relative humidity $30-50 \%$, air pressure 1014-1032 mbar.

Motion mode is incremental exercise tests in s vehicle. Subjects have 5 minutes for preparatory activities with $60 \mathrm{~W}$ to suit the load and bike speed, and then do exercise test according to the set load. The experiments collect exhaled breath and record heart rate in the testing process [6-8].

\subsection{Experiment Scheme}

Experiments select four different diets: Diet CC(high sugar diet and sugary drinks), Diet FC(high fat diet and sugary drinks), Diet CP(high sugar diet and polysaccharide peptide drink) and Diet FP(high fat diet and polysaccharide peptide drink). They contain the equal amount of food. Subjects should intake the same food as usual for the lunch and dinner before the experiment. They are dominated by bland foods and avoid eating fatty foods $[9,10]$.

The experiment uses self-control and balance in overlapping to test subjects which is conducted four times. Each test interval of at least 2 times is 7 days in order to eliminate the effects of previous tests. Subjects were divided into 4 teams, and there are 2 subjects every day from first week to the fourth week as shown in Table 2. The two subjects must eat the same food every day. Feeding sequence is as follows:

\subsection{Detection Index}

A series of indicators need to be tested. (1) blood glucose. We use test paper method to measure fingertip blood. (2) Serum hormone. Insulin was measured by chemiluminescence method. Leptin was measured by ELISA method. Relation of standard curve is as follows:

$$
Y=1.6964 X_{2}-19.464 X+55.179\left(R^{2}=0.9737\right)
$$

(3) Metabolic markers. There is a series of breathing gas metabolism, including respiratory quotient, energy consumption, $\mathrm{CHO}$ oxidation rate, $\mathrm{FAT}$ oxidation rate, $\mathrm{CHO} \%$, $\mathrm{FAT} \%$ and so on. We use the following formula to estimate the total $\mathrm{CHO}$ and total fat oxidation speed.

$$
\begin{aligned}
& \mathrm{CHO}=4.585 \times \mathrm{VCO}_{2}-3.226 \times \mathrm{VO}_{2} \\
& F A T=1.695 \times \mathrm{VO}_{2}-1.701 \times \mathrm{VCO}_{2}
\end{aligned}
$$

\subsection{Statistical Analysis}

Experimental data is indicated by mean plus standard error. Statistical analysis is completed by SPSS software. Firstly, we use K-S method to examine if the indicators data are normally distributed, and then parametric statistical analysis. Secondly, we define two variables corresponding to two factors in this study. We build a 2 X2 two factor single variable database according to levels of each factor. Generalized linear model is used for a single dependent variable multi-factor analysis of variance in order to analyze interaction effects between these two factors of high-fat and skin supplementation. We use GLM multivariate variance analysis to test differences between the dependent variable and multiple dependent variables on the same point in time. Significant test results take for $\mathrm{P}<0.05$ and insignificant test results take for $\mathrm{P}<0.01$.

\section{EXPERIMENTAL RESULTS}

\subsection{Food Intake Results}

Drink energy ingredient is as follows in Table $\mathbf{3}$.

The nutritional content of food is as follows in Table 4.

\subsection{Effect of Different Diet on Glucose Metabolism- Related Indicators}

We have measured the glucose after 45 minutes of meals. The CC group is significantly higher than group of FC, CP and FP and it has significantly differences $(\mathrm{P}<0.05)$. Analysis results showed that the main effect of polysaccharide on glucose has statistics meaning at the moment $(\mathrm{F}=5.601, \mathrm{P}=0.026)$. And main effect of high fat on glucose also has statistics meaning $(\mathrm{F}=13.336, \mathrm{P}=0.001)$, 
Table 3. Specific energies of the drink ingredients $(100 \mathrm{ml})$.

\begin{tabular}{|c|c|c|}
\hline & Polysaccharide peptide drink & Sugary drinks \\
\hline Energy(Kcal) & 31 & $>19$ \\
\hline $\mathrm{CHO}(\mathrm{g})$ & 6 & $>5$ \\
\hline $\operatorname{Pro}(\mathrm{g})$ & $>1$ & 0 \\
\hline
\end{tabular}

Table 4. Nutritional content of food.

\begin{tabular}{|c|c|c|c|c|}
\hline & CC & FC & CP & 1181.83 \\
\hline Energy & 704.81 & 1134.88 & 719.47 & 20.93 \\
\hline PRO & 25.19 & 14.93 & $16.59 \%$ & $7.08 \%$ \\
$\%$ & $14.29 \%$ & $5.23 \%$ & 108.77 & 101.69 \\
\hline CHO & 114.76 & 101.69 & $61.07 \%$ & $34.42 \%$ \\
\hline FAT & $65.14 \%$ & $35.84 \%$ & 16.77 & 74.94 \\
\hline Water & 16.77 & 74.94 & $20.08 \%$ & $57.07 \%$ \\
\hline Ash & $21.41 \%$ & $59.43 \%$ & 897.99 & 815.36 \\
\hline
\end{tabular}

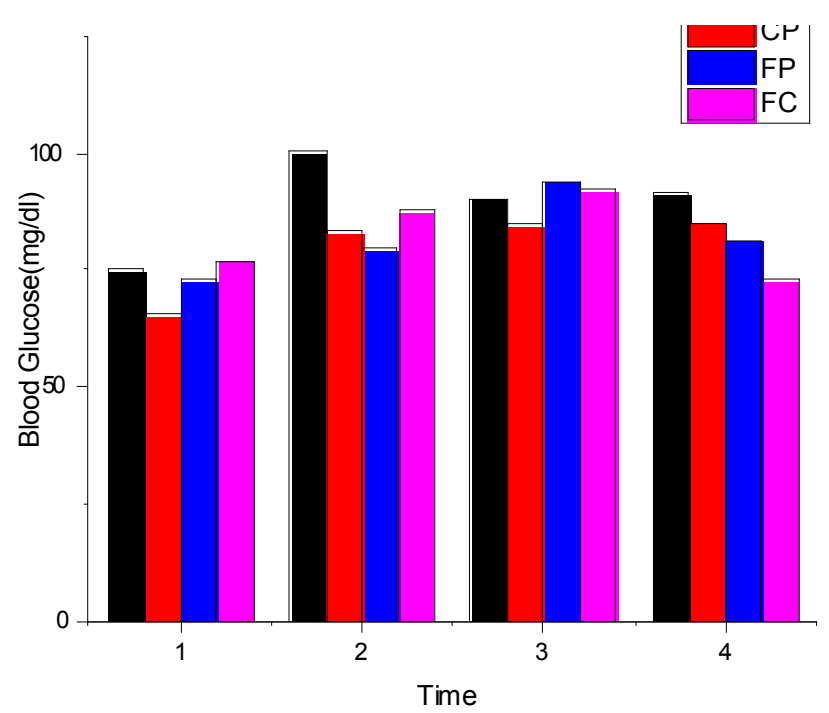

Fig. (1). Effect of aerobic exercise and different diets on blood glucose levels.

but they have no interactive effect. On the other hand, we have measured the glucose after 45 minutes of sports. The $\mathrm{CC}$ group is significantly higher than group of FC. Analysis results showed that the main effect of high fat on glucose also has statistics meaning $(\mathrm{F}=5.386, \mathrm{P}=0.028)$ Fig. (1).

Group of $\mathrm{CC}$ and $\mathrm{CP}$ have no significant change on serum TG levels in the experiment. Serum TG of group of FC and FP trends similar. Serum TG levels is not significantly increased after meals, but were significantly higher than in the early morning quiet time after exercise

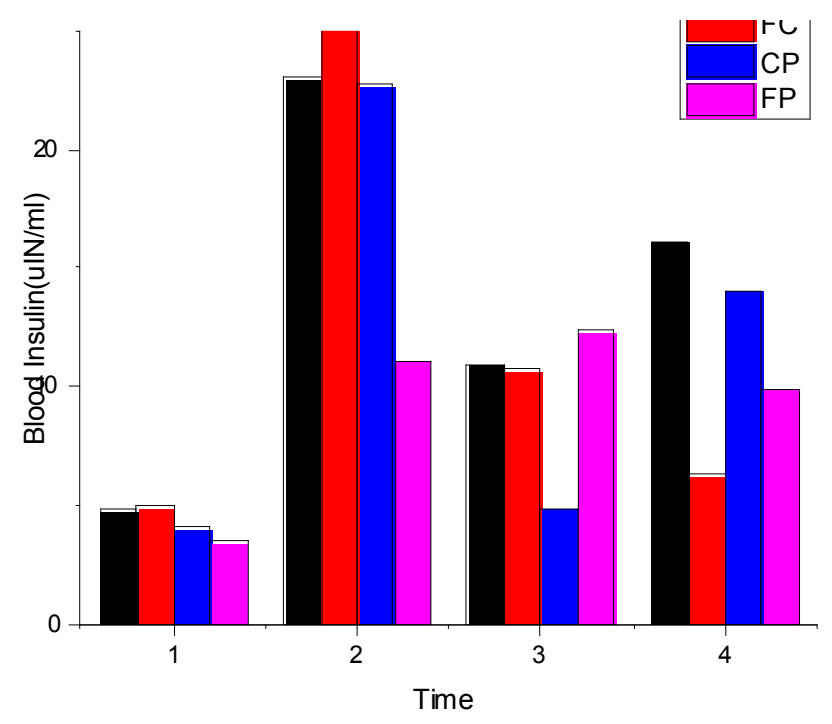

Fig. (2). Effects of different diet and aerobic exercise on serum insulin.

$(\mathrm{P}<0.05)$. TG levels were significantly higher as compared to the early morning quiet time and after meals when exercise of 45 minutes $(\mathrm{P}<0.01)$. Serum TG levels is not significantly increased after meals in group of $\mathrm{PF}$, but were significantly higher than in the early morning quiet time after exercise $(\mathrm{P}<0.01)$ and also were significantly higher than after meals Fig. (2).

The TG levels in group of CC and FC differ significantly after exercise for 45 minutes $(\mathrm{P}<0.05)$, and were significantly different with group of FP $(\mathrm{P}<0.01)$. The TG levels in group of FP and CP differ significantly $(\mathrm{P}<0.05)$, and were significantly different with group of $\mathrm{CC}(\mathrm{P}<0.01)$. 


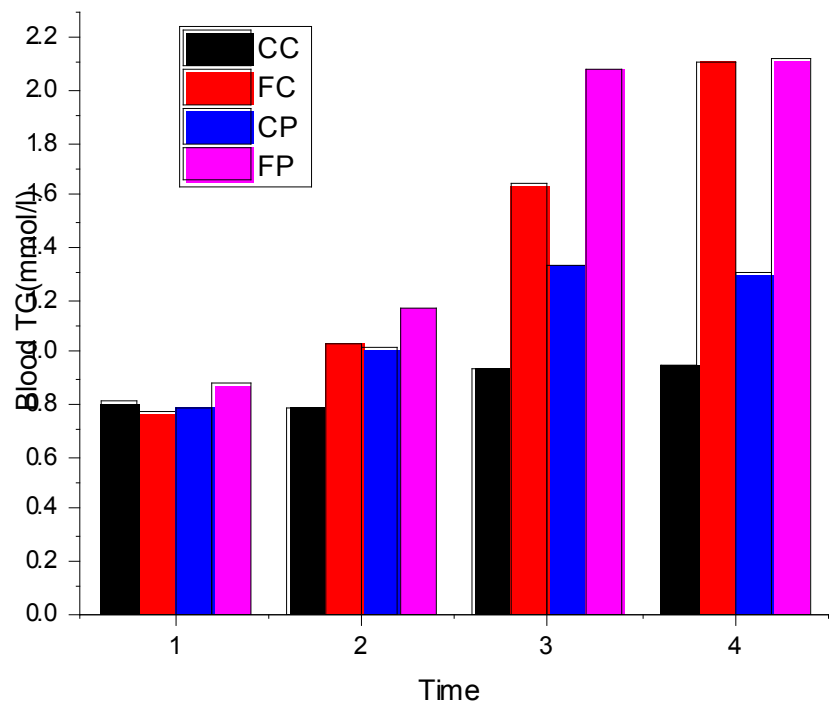

Fig. (3). Effects of different diet and aerobic exercise on serum TG.

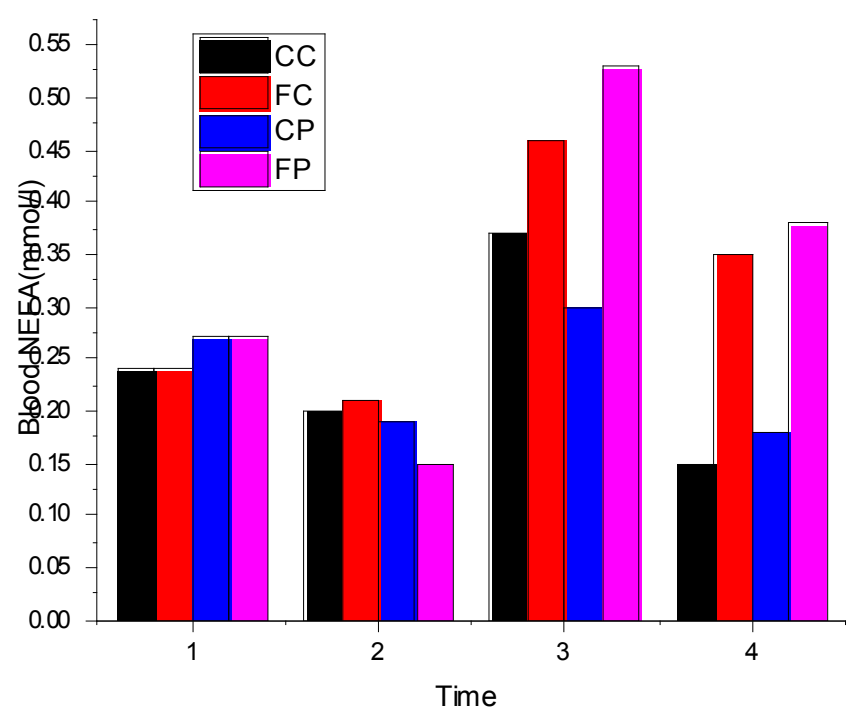

Fig. (4). Effects of different diet and aerobic exercise on serum NEFA.

Analysis results showed that main effect of high lipid on serum TG level was statistically significant $(\mathrm{F}=11.281$, $\mathrm{P}=0.002$ ) as shown in Fig. (3).

\subsection{Discussion and Analysis}

Theoretically speaking, the activity when you take sugar diet or soda before exercise can cause hypoglycemia in sports. Research has also proved that eating at this time will have a negative effect on their scores. This experiment observed groups of CC and CP are high in blood sugar and insulin levels after exercise for 45 minutes which will help promote the glycogen recovery after exercise.

Studies have shown that metabolic status also affects the rate of fat oxidation in addition to the impact of the type of fatty acids. Hunger and chronic exercise conditions can also lead to increased lipolysis and oxidation. Glucose and insulin levels can inhibit the oxidation of fatty acids on substrates and hormones Fig. (4).

\section{CONCLUSION}

In this paper, we can get some results. (1) RQ will be reduced after high fat diet and will increase after high sugar diet. RQ after high sugar diet will rise more than after high fat diet. (2) High sugar diet may improve glucose oxidation rate and proportion of energy supply. High fat diet may improve fat oxidation rate and proportion of energy supply. After the intake of the same high-fat diet and high sugar diet, dietary energy intake of high fat diet is higher than high sugar diet. But energy expenditure did not increase after a high fat diet which can lead to the body's energy balance. (3) Candy skin drinks can promote fat oxidation during and after exercise after high fat diet.

It is desirable to further apply the analysis results to solving those more complex real-world health problems and it will be our further work.

\section{CONFLICT OF INTEREST}

The author confirms that this article content has no conflict of interest.

\section{ACKNOWLEDGEMENTS}

This work is supported by the Key Project of Guangxi Social Sciences, China (No. gxsk201424), the Education Science fund of the Education Department of Guangxi, China (No. 2014JGA268), and Guangxi Office for Education Sciences Planning, China (No. 2013C108).

\section{REFERENCES}

[1] P. D. Cani, R. Bibiloni, C. Knauf, A. Waget, and A. M. Neyrinck, Nathalie M. Delzenne1, and Re'my Burcelin "Changes in gut micro biota control metabolic end toxemia-induced inflammation in high-fat diet-induced obesity and diabetes in mice," Diabetes, vol. 57, no.6, pp. 1470-1481, 2008.

[2] P. D. Cani, A. M. Neyrinck, F. Fava, C. Knauf, R.G. Burcelin K.M. Tuohy, G.R. Gibson, and N.M. Delzenne, "Selective increases obifid bacteria in gut micro flora improve high-fat-diet-induced diabetes in mice through a mechanism associated with endotoxaemia," Diabetologia, vol. 50, no.11, pp. 2374-2383, 2007.

[3] F. Gomez, E. Jequier, and V. Chabot,, "Carbohydrate and lipid oxidation in normal human subjects: its influence on glucose tolerance and insulin response to glucose," Metabolism, vol. 21, no.5, pp. 381-391, 1972 .

[4] Y. Jimenez-Gomez, J. A. Mattison, and K. J. Pearson, "Resveratrol improves adipose insulin signaling and reduces the inflammatory response in adipose tissue of rhesus monkeys on high-fat, highsugar diet," Cell metabolism, vol. 18, no.4, pp. 533-545, 2013.

[5] L. E. Kuo, M. Czarnecka, and J. B. Kitlinska, "Chronic stress, combined with a high - fat/high - sugar diet, shifts sympathetic signaling toward neuropeptide $\mathrm{Y}$ and leads to obesity and the metabolic syndrome," Annals of the New York Academy of Sciences, vol. 1148, no.1, pp. 232-237, 2008.

[6] N. Kubota, Y. Terauchi, and H. Miki, "PPARy mediates high-fat diet-induced adipocyte hypertrophy and insulin resistance," Molecular cell, vol. 4, no.4, pp. 597-609, 1994.

[7] X. F. Li, W. B. Liu, and K. L. Lu, "Dietary carbohydrate/lipid ratios affect stress, oxidative status and non-specific immune responses of fingerling blunt snout bream, Megalobrama amblycephala," Fish \& shellfish immunology, vol. 33, no.2, pp. 316-323, 2012. 
[8] L. P. Musselman, J. L. Fink, and K. Narzinski, "A high-sugar diet [1] produces obesity and insulin resistance in wild-type Drosophila," Disease models \& mechanisms, vol. 4, no.6, pp. 842-849, 2011.
[9] J.A. Yanovski, and S.Z. Yanovski, "Recent advances in basic obesity research," vol. 282, pp.1504-1506, 1999.

[10] J. Yudkin, "The treatment of obesity by the "high-fat" diet," The inevitability of calories, Lancet, vol. 2, pp. 939-941, 1960.

Received: June 10, 2015

Revised: July 29, 2015

Accepted: August 15, 2015

(C) Ronghua Liu; Licensee Bentham Open.

This is an open access article licensed under the terms of the (https://creativecommons.org/licenses/by/4.0/legalcode), which permits unrestricted, noncommercial use, distribution and reproduction in any medium, provided the work is properly cited. 